\title{
Harmonic Progression in Bioinformatics and Recurrent Series in Inherited Biostructures
}

\author{
Vladimir Verevkin, Sergey Petoukhov* \\ Mechanical Engineering Research Institute of RAS, RU-101990, Moscow, Russia
}

\begin{abstract}
The a rticle is de voted to the s tudy of ne w approaches t o the d evelopment of mathematical models in genetic biomechanics, which studies the structural relationships of the genetic coding system with genetically inherited biological forms. More specifically, we are talking about models based on the recurrent harmonic progression whose connection with the information sequences of DNA molecules in the genomes of higher and lower organisms was recently revealed. In particular, the article describes previously unknown connections of the function of natural logarithms with the structures of the molecular genetic system, which allow modelling th e $\mathrm{m}$ ain $\mathrm{p}$ sychophysical lo garithmic la w b y W eber-Fechner a nd a lso $\mathrm{m}$ any ot her logarithmic structures in genetically in herited biological systems. In physics, the harmonic progression is traditionally c onsidered, first of all, as related to standing waves in resonators. Our results a re correlated with Frohlich's vibration-resonant theory about collective quantum effects and long-range co mmunication in biological systems.
\end{abstract}

\section{Introduction}

Investigations o fs ymmetries in in herited b iological structures are an important component of biomechanics, biophysics, bi ochemistry, a nd ot her s ciences of living matter. Many monographs, international forums, including the Nobel Symposium on Symmetries, etc. are devoted to them. The continuation of these studies is an urgent ta sk, which is a ssociated with the $d$ isclosure of many s ecrets of 1 iving $n$ ature an $d t$ he creation of $n$ ew biotechnologies.

In particular, these studies have long drawn attention to $t$ he r ecurrent $s$ tructure of $\mathrm{m}$ any i nherited $\mathrm{b}$ iological configurations, which co nsist o f $\mathrm{r}$ ecurrently $\mathrm{r}$ epeating blocks $r$ elated, f or e xample, b y s imilarity s ymmetries; these co nfigurations ar e ab undant in flowers, $s$ hells of mollusks, metameric b odies of an imal o rganisms, et c. [1]. The inheritance of configurations relies on the work of $t$ he $g$ enetic c oding s ystem, which a llows $t$ he reproduction of $\mathrm{s}$ uch forms in a s eries of generations. Recently, in the genomes of higher and lower organisms, an important rule for the recurrent organization of DNA nucleotide sequences was $d$ iscovered; t hese s equences serve for the transfering genetic information $[2,3]$. This hyperbolic rule for the relationship of oligomer sums is associated with a long-known harmonic progression (1):

$$
1 / 1,1 / 2,1 / 3,1 / 4, \ldots, 1 / n
$$

The use of properties of this progression allows building new approches and models in genetic biomechanics, algebraic biology and biotechnologies.

\section{Computanional approaches, models}

Our computational approaches and models of biological structures are based on the mathematical properties of the harmonic progression (1). Its historically renowned name - "harmonic progression" - derives from its association with a series of harmonics in music (or with a series of standing wavelengths in a vibrating string). It is also indirectly associated with a set of trigonometric functions in the Fourier series. Pythagoras, Orem, Leibniz, Newton, Euler, Fourier, Dirichlet, Riemann and many others were engaged in research of mathematical properties, generalizations and applications of the harmonic progression in various problems. The unexpected discovery of this recurrent harmonic progression in genomes, which are the genetic information basis of living organisms, entails many new scientific issues. The aim and objectives of this study are to study how the mathematical properties of this recurrent genetic progression (1) are able to influence the inherited biomechanical structures bearing the stamp of the structural features of genetic coding. This study leads to the emergence of new model approaches in genetic biomechanics, developing the theme of structural relationships between genetic coding and inherited biomechanical phenomena.

Organisms are cooperative aggregates of a huge number of various molecules that have the ability to reproduce themself in their descendants. Gr. Mendel in experiments on crossing organisms showed that the inheritance of traits to descendants obeys certain algebraic rules. Thus, he actually became the founder of

Corresponding author: spetoukhov@gmail.com 
algebraic biology, which uses algebraic methods to model inherited biological phenomena. At present, in this regard, an international interdisciplinary seminar "Algebraic biology and systems theory" (https://www.youtube.com/channel/UC8JLsuRzzPsRiH wrwEjMCtw ) is functioning in Moscow. According to Mendel's law of independent inheritance of traits, information from the level of genetic DNA molecules dictates the macrostructure of living bodies through many independent channels, despite strong noises and interference. For example, hair, eye, and skin colors are inherited independently of each other. This determinism is provided by unknown algorithms of multichannel noise-immune coding. Accordingly, each organism is a multichannel noise-immune coding machine. Our studies of the properties of genetic inheritance of biological structures and the possibilities of their algebraic modeling are based on the well-known mathematical formalisms of matrix and tensor analysis, recurrent sequences, the theory of information noise-immune coding, as well as on known academic information about genetic and other biological structures.

\section{Results and discussion}

Let us present some of the possibilities we have studied of us ing $t$ he mathematical $p$ roperties o $f h$ armonic progression ( 1), which $\mathrm{i}$ s a ssociated with $\mathrm{t}$ he organization of genomes of higher and lower organisms, for modeling $\mathrm{i}$ nherited $\mathrm{b}$ iological $\mathrm{s}$ tructures a nd processes.

Harmonic $\mathrm{p}$ rogression is $\mathrm{r}$ elated to the w ell-known concept of harmonic mean and is a $\mathrm{r}$ ecurrent s equence based on $i \mathrm{t}$ : $\mathrm{k}$ nowing $\mathrm{t}$ wo a djacent $\mathrm{m}$ embers of $\mathrm{t}$ his progression, you can build the entire given progression. Indeed, the harmonic mean $x_{n+1}$ for two numbers $x_{n}$ and $x_{n+2}$ is expressed by the relation (2):

$$
x_{n+1}=\left(\left(x_{n}^{-1}+x_{n+2}{ }^{-1}\right) / 2\right)^{-1}=2 x_{n} x_{n+2} /\left(x_{n}+x_{n+2}\right)
$$

Knowing t wo neighboring num bers $x_{n}$ and $x_{n+1}$ in this tr iplet o f numbers, the th ird number $x_{n+2}$ is determined, respectively, by the relation (3):

$$
x_{n+2} \quad=\quad x_{n} x_{n+1} \quad /\left(2 x_{n} \quad-\quad x_{n+1}\right)
$$

Successively applying this recurrence relation (3) to the $\mathrm{f}$ irst te rms $x_{1}=1$ an $\mathrm{d} x_{2}=1 / 2$ of $\mathrm{t}$ he ha rmonic progression (1), we obtain the entire series of its terms. It seems interesting and $\mathrm{u}$ seful to investigate in the future the harmonic mean ratios in inherited b iological structures and processes.

Pairs of numbers $n$ and $1 / n$ define points on the plane with c oordinates $[n, 1 / n]$ in th e Cartesian c oordinate system be longing t o t he hyperbola $\mathrm{y}=1 / n$, which is closely r elated to the natural logarithm function $\ln (x)$. As y ou know, the natural logarithm can be defined for any pos itive $\mathrm{r}$ eal num ber $a>1$ as the ar ea $u$ nder $\mathrm{t}$ he hyperbola $y=1 / x$ from 1 to $a$ (Fig. 1) [4].

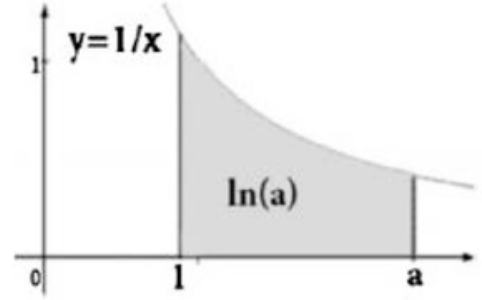

Fig. 1. Illustration of the natural logarithm $\ln (\mathrm{x})$ as the area under the hyperbola $\mathrm{y}=1 / \mathrm{x}$.

The natural logarithm function is widely used in mathematical biology to model inherited biological phenomena. For example, various types of inherited sensory perceptions - sight, hearing, smell, tactile sense, taste - are subject to the basic psychophysical WeberFechner law, which is logarithmic. This law states that the intensity of perception is proportional to the logarithm of the intensity of the stimulus. By virtue of this law, for example, the power of sound in engineering is expressed in a logarithmic decibel scale. The logarithmic nature of perception provides the body with a huge expansion of the range of perceived stimuli, which can differ in intensity by many millions or even billions of times: for example, hammer strikes on a steel plate generate noise that is a hundred billion times louder than the quiet rustle of leaves, and the brightness of a volt arc trillions of times the brightness of a faint star, barely visible in the night sky [5]. It can be assumed that organisms use natural logarithms as the basis for their internal counting.

Taking into account the named connection of the natural logarithm function with the hyperbola $y=1 / x$, we note that the recurrent harmonic progression (1) is conjugate, respectively, with a recurrent sequence of natural logarithms (4):

$$
\ln (1), \ln (2), \ln (3), \ldots, \ln (\mathrm{n})
$$

Fig. 2 shows this recurrent sequence (4) in graphical form i n a p olar co ordinate s ystem, where a $\mathrm{n}$ i nteger positive $\mathrm{v}$ ariable $n$ plays the $\mathrm{r}$ ole $\mathrm{o} \mathrm{ft}$ he a ngular coordinate $\theta$, a nd $\mathrm{ln}(n) \mathrm{p}$ lays th e r ole of th e $\mathrm{r}$ adius vector $r$.

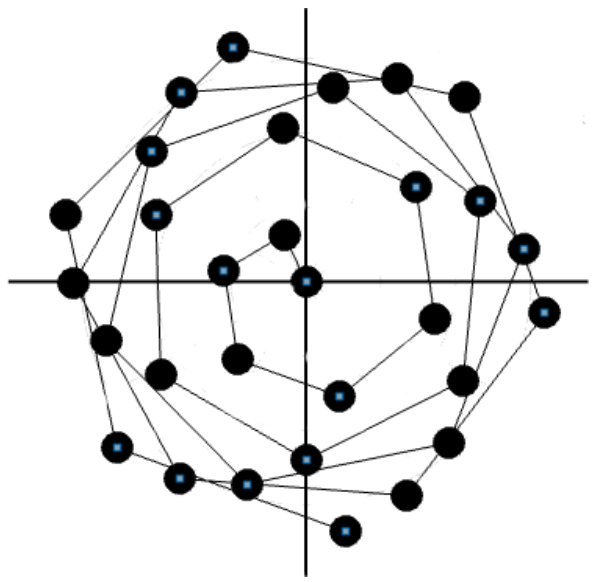

Fig. 2. The graph of a sequence of discrete values of the natural $\operatorname{logarithm} \ln (\mathrm{n})$ in a polar coordinat system for $\mathrm{n}=1,2, \ldots, 30$. 
Turning to discrete values of functions in modeling biological phenomena seems to be fundamentally important due to the discrete nature of the organization of both the genetic coding system (discrete DNA alphabets, etc.) and a variety of biomechanical processes (for example, the seemingly continuous movement of a human hand is provided due to the total effect of discrete contractions of a huge variety of muscle units, each of which has its own threshold of excitability and is contracted out of step with the rest). As is known, for all excitable cells of the body, the genetically inherited universal law of "all or nothing" of a discrete nature is valid: the cell does not respond at all to stimuli of a subthreshold value, but reacts to above-threshold stimuli with full amplitude. These facts indicate that biomechanics, if it takes into account these fundamental features of living tissue, should be developed as the mechanics of discrete sequences and processes, taking into account their relationship with discrete genetic coding.

The use of a polar coordinate system for the graphic representation of biological structures as configurations of a spiral type is justified by the fact that spirals have long been considered as "the curves of life" $[6,7]$ : spiral and helical configurations are characteristic of DNA molecules, proteins, bones, tendons, muscles, etc. etc. For example, the heart is a holistic spiral muscle that pushes blood by its action of twisting and untwisting, similar to how a washerwoman squeezes water out of laundry by twisting it. Spiral motions ( nutation) a re observed during the growth of roots and shoots, tendrils of plants are spirally wrapped, a tissue in the trunks of trees $g$ rows spirally, e tc. B ecause o f spiral configurations, all the fluids in the body (blood, lymph, and urine) are spiral.

Spirals and helices in living matter are quite different from spirals and helices in inanimate nature:

1. They ar e g enetically $\mathrm{i}$ nherited $\mathrm{i} \mathrm{nt}$ he $\mathrm{c}$ hain o $\mathrm{f}$ generations and are as sociated with structures of a molecular-genetic system that is full of spirals and super spirals at different levels of it s organization, from DNA to chromosomes;

2. Characteristics of b iological spirals are as sociated with Fibonacci numbers at different levels and branches of the b iological e volution, from a lphapeptides to the s tructure of t he integral b odies of plants and animals, which is reflected in the famous bio-mathematical la ws o f $p$ hyllotaxis, to which thousands of pu blications have be en de voted worldwide [8].

The a mounts of the initial members of the harmonic progression (1) are termed harmonic numbers $\mathrm{H}_{n}$. These numbers a ppear s o of ten in the a nalysis of a lgorithms that computer scientists need a special notation for them. The series (5) presents first harmonic numbers $\mathrm{H}_{n}$ :

$$
1,3 / 2,11 / 6,25 / 12,137 / 60, \ldots
$$

Harmonic numbers are related to the harmonic mean in th at th e $n$-th harmonic $\mathrm{n}$ umber $\mathrm{i}$ s al so $n$ times th $\mathrm{e}$ reciprocal of the $h$ armonic $m$ ean of the first $n$ positive integers $1,2,3, \ldots, n$.
Harmonic n umbers are co nnected with the function of $\mathrm{n}$ atural lo garithm: a $\mathrm{s}$ it is $\mathrm{k}$ nown, th e harmonic number $\mathrm{H}_{n}$ is $\mathrm{t}$ he $\mathrm{d}$ iscrete analog o $\mathrm{ft}$ he co ntinuous function $1 \mathrm{n}(x)$. F ig. 3 shows a $\mathrm{n}$ a pproximation of $\mathrm{t}$ he function of the natural logarithm in Fig. 3 by the sum of areas $1,1 / 2,1 / 3,1 / 4, \ldots, 1 / n$ of the shown rectangles; sums of these areas form the series of harmonic numbers $\mathrm{H}_{n}$ (4).

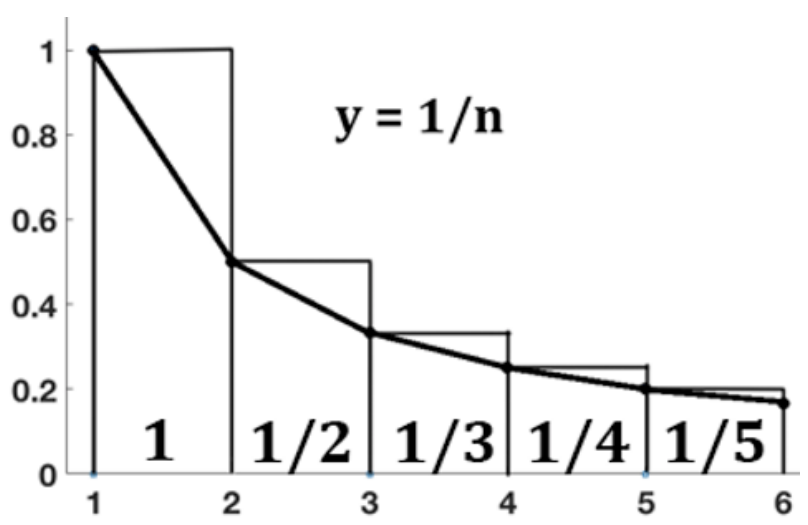

Fig. 3. An approximation of the area under the hyperbola $y=$ $1 / \mathrm{x}$ by the sum of areas $1,1 / 2,1 / 3,1 / 4, \ldots, 1 / \mathrm{n}$ of the shown rectangles, whose areas form the series of harmonic numbers Hn (4).

In 1740, Euler obtained an asymptotic expression for the harmonic numbers $\mathrm{H}_{n}$ :

$$
\mathrm{H}_{n}=\ln (n)+\gamma+\varepsilon
$$

where $\gamma=0.5772 \ldots$ is the Euler-Mascheroni co nstant, and the value $\varepsilon$ tends to zer ow ith i ncreasing $n$. Therefore, with increasing $n$, the harmonic number $\mathrm{H}_{n}$ is more and more accurately expressed by the sum (6):

$$
\mathrm{H}_{n} \approx \ln (n)+0.5772=\ln (n)+\ln (1.7810)
$$

To improve an approximation of the logarithmic function $\ln (x)$ by the total area of the rectangles (Fig. 4), you can c onsider - instead of the classic harmonic progression $1,2, \ldots, 1 / k$ - more fractional s equences, called $t$ he " compressed $h$ armonic $p$ rogression" ( or briefly, the C-harmonic progression):

$$
1,1+1 / m, 1+2 / m, \ldots, 1+k / m,
$$

where the additional factor $m$ can take integer values 1 , $2,3, \ldots$. The a mounts of the initial members of the $\mathrm{C}$ harmonic $\mathrm{p}$ rogression ( 8 ) a re $\mathrm{t}$ ermed b y au thors as "compressed h armonic numbers" ( or C-harmonic numbers). $\mathrm{F}$ ig. $4 \mathrm{~s}$ hows a $\mathrm{n}$ i mproved a pproximation of the continuous function $\ln (x)$ b y $\mathrm{C}$-harmonic nu mbers $\mathrm{H}_{n}{ }^{[m]}$ at $m=3$ : the area under the hyperbola, representing the natural logarithm, is better approximated by the total area of the rectangles equal to the $\mathrm{C}$-harmonic nu mber $\mathrm{H}_{n}{ }^{[3]}$. $\mathrm{T}$ he 1 arger $\mathrm{t}$ he va lue $m, \mathrm{t}$ he $\mathrm{b}$ etter $\mathrm{t}$ he approximation o $\mathrm{ft}$ he continuous function $1 \mathrm{n}(x) \mathrm{b} \mathrm{y}$ discrete C-harmonic numbers $\mathrm{H}_{n}{ }^{[\mathrm{m}]}$ is provided. 


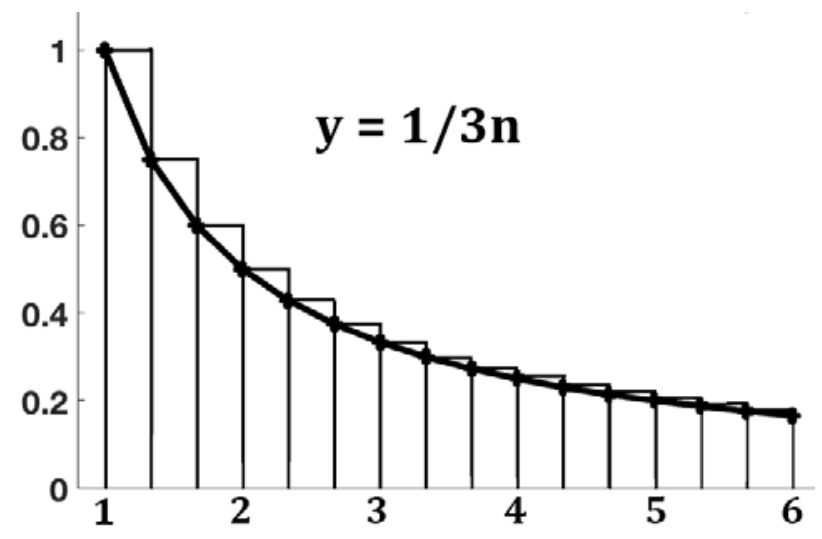

Fig. 4. The approximations of continuous function $\ln (\mathrm{x})$, by compressed harmonic numbers $\mathrm{Hn}[\mathrm{m}]$ under $\mathrm{m}=3$.

The na tural logarithm function $y=\ln (x)$, which is important for modeling inherited biological phenomena, is mutually inverse to the exponent function $x=\exp (y)$, which a re a lso $\mathrm{u}$ sed $\mathrm{f}$ or a $\mathrm{l}$ ong $\mathrm{t}$ ime $\mathrm{t} \mathrm{o}$ model morphogenetic a nd ot her bi o-phenomena. A ccordingly, the $r$ ecurrent s equence (4) is mutually i nverse to th $\mathrm{e}$ recurrent sequence (9) of exponentials $\exp (n)$ :

$$
\exp (1), \exp (2), \exp (3), \ldots, \exp (n)
$$

Fig. 5 shows a continuous exponential function $\exp (x)$ and a discrete recurrent exponential sequence in a polar coordinate system. These graphs correspond to continuous and discrete logarithmic spirals.
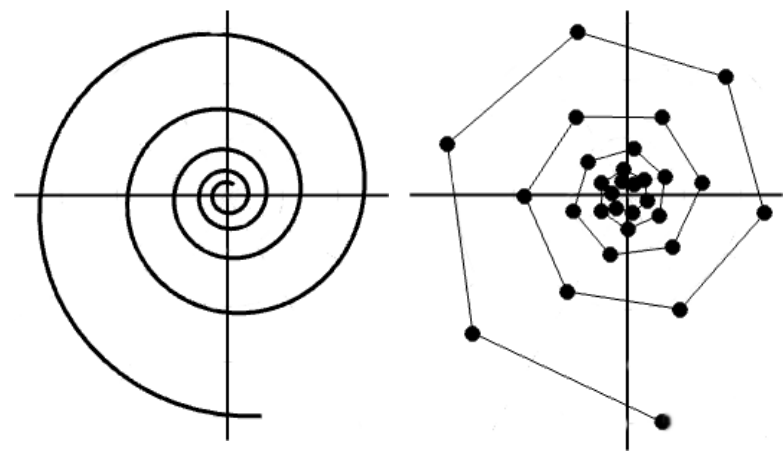

Fig. 5. Graphs of a continuous exponential function $\exp (0.1 \mathrm{x})$ and discrete sequence exp $(0.1 \mathrm{n})$ for $\mathrm{n}=1,2, \ldots, 30$.

Logarithmic functions and logarithmic spirals model many genetically inherited biological phenomena, including morphogenetic configurations of phyllotaxis laws [8], which are associated with another recurrent sequence - a series of Fibonacci numbers related to the golden ratio.

Let us add that many cases of biomechanical movements - growth and motor - of living bodies can be modeled as a transition from one discrete recurrent logarithmic spiral to another. This corresponds to the statement of the classic of biomechanics N.A. Bernstein: "The idea that movement is in many respects like an organ seems extremely fruitful" [9].

Mendel discovered in his experiments on the crossing of organisms that the inheritance of their traits occurs according to algebraic rules, despite the colossal complexity and heterogeneity of the molecular structure of living bodies. In this regard, he can be considered the founder of algebraic biology. We believe that algebraic biology is connected with quantum biology, whose history is presented in [10]. The human adult body contains about 100 trillion cells, the ensemble of which behaves as a whole. It seems natural to make a consideration of such complex ensembles from the standpoint of quantum mechanics, to which the carriers of genetic information - DNA molecules - belong.

An important step in the development of quantum biology, to which our study of genetically inherited biological structures belong, was the creation of the theory by H. Frohlich for collective quantum effects in biological systems $[11,12]$. This theory testifies in favor that living bodies are a kind of analog of quantum BoseEinstein condensate. Nobel laureate R. Penrose uses Frolich's theory to substantiate his ideas about the brain as a quantum computer. He writes about it in his book [13, pp. 352 and 367]: «The distinguished physicist Herbert Frohlich suggested a possible role for collective quantum effects in biological systems. ... Frohlich was led to propose, in 1968, that there should be vibrational effects within active cells, which would resonate with microwave electromagnetic radiation, at $1011 \mathrm{~Hz}$, as a result of a biological quantum coherence phenomenon. Instead of needing a low temperature, the effects arise from the existence of a large energy of metabolic drive. There is now some respectable observational evidence, in many biological systems, for precisely the kind of effect that Frohlich had predicted in 1968 ... He argued that so long as the energy of metabolic drive is large enough, and the dielectric properties of the materials concerned are sufficiently extreme, then there is the possibility of large-scale quantum coherence similar to that which occurs in the phenomena of superconductivity and superfluidity - sometimes referred to as BoseEinstein condensation - even at the relatively high temperatures that are present in biological systems... In a Bose-Einstein condensate, large numbers of particles participate collectively in a single quantum state... There is a coherence on a large scale, where many of the strange features of quantum wavefunctions hold at a macroscopic level".

According to Fröhlich, under appropriate conditions a phenomenon quite similar to a Bose condensation may occur in substances, which possess longitudinal electric modes. If energy is fed into these modes and thence transferred to other degrees of freedom of the substance, then a stationary state will be reached in which the energy content of the electric modes is larger than in the thermal equilibrium. This excess energy is found to be channelled into a single mode - exactly as in Bose condensation - provided the energy supply exceeds a critical value. Under these circumstances a random supply of energy is thus not completely thermalized but partly used in maintaining a coherent electric wave in the substance. This theory is supported by many authors, for example [14-17].

Our results about hyperbolic rules of cooperative genomic organization connected with the harmonic progression, which is known in physics of standing waves in resonators, are correlated with ideas of the 
article "Genetic code as Language" (see in [11]). It was written by F.Frohlich, the son of H. Fröhlich. This article was devoted to quantum coherence states and long-range communication in genomes. Its author noted that the genome as a whole must contain some sort of longdistance communication. Using H. Frohlich's hypothesis, he wrote that long-range coherent vibrations will lead to resonance between a differentiated cell with its own characteristic vibrations and the chromosome such that the chromosome-particular region responding to this characteristic frequency will be activated or opened up so it can produce the appropriate proteins. Such a resonance could transport the embryological, already partially induced cells to their target and there they would be further fixed into producing the correct proteins for this organ through superimposed resonance and why i f it were to o m ature, it w ould $\mathrm{n}$ ot s o a dapt; there might be degrees of resonance. The conception of multi-resonance genetics was represented also in [18].

\section{Some concluding remarks}

The work of the laboratory for the research of biomechanical systems of the IMASH RAS (http://eng.imash.ru/) revealed fundamental hyperbolic rules for the organization of DNA information texts in the genomes of higher and lower organisms (eukaryotes and prokaryotes), associated with harmonic progression (1), which is well-known in science in connection with various mathematical and physical problems. The discovery of the important role of the harmonic progression in genetic informatics leads to the development of new mathematical models in genetic biomechanics, which studies the relationship of inherited biomechanical structures with genetic DNA texts, since DNA molecules play a dictatorial role in living organisms, determining their genetically inherited physiological structures [10]. Additionally, we note that harmonic progression plays a prominent role in aesthetics. This progression (1) is important not only for music with its systems of musical harmony and musical overtones. At least since the time of the Pythagorean doctrine of the aesthetics of proportions, there has been an idea that the composer R. Schumann expressed as follows: "The aesthetics of one art is at the same time the aesthetics of another art; only the material is different" [19]. In light of this, architecture has long been interpreted as frozen music and music as dynamic architecture. In connection with our research, assumptions arise about the genetic basis of a number of aesthetic parallels in various arts.

The above features of harmonic progression (1) do not exhaust its rich mathematical features, which can serve to create new approaches to the construction and substantiation of models in genetic biomechanics, as well as contribute to the development of algebraic and quantum biology [20].

\section{Conclusions}

The present study leads us to conclude that
(1) The discovery of the connection of cooperative genomic o rganization with $\mathrm{t}$ he recurrent harmonic progression (1) gives new a pproaches f or de veloping mathematical models of genetically i nherited biological structures and also for searching hidden genetic rules;

(2) The connection of the genomic harmonic progression with functions of natural logarithms gives us a new $u$ nderstanding the main $p$ sychophysical $l a w b y$ Weber-Fechner an do ther $g$ enetically $i$ nherited logarithmic structures in living bodies;

(3) The $r$ eceived $r$ esults ar e correlated $w$ ith Frohlich's vibration-resonant theory about collective quantum ef fects an d l ong-range co mmunication in biological systems.

The authors are grateful to G. Darvas, E. Fimmel, V. Svirin, I. Stepanyan, and G. Tolokonnikov for assistance in the work and discussion of the results.

\section{References}

1. A.V.S hubnikov, V .A. K optsik, Symmetry in Science and Art, 3rd ed. (RChD, Moscow, 2004)

2. S.V. Petoukhov, Preprints 2020, 2020050471 (2020)

3. S.V. Petoukhov, Biosystems, 198, 104273 (2020)

4. J.H. C onway, R.K. G uy, The Book of Number (Copernicus, N.-Y., 1995)

5. N. Ya . Vi lenkin, Functions in nature and technology (Prosveschenie, M oscow, 1985) [in Russian]

6. T.A. Cook, The Curves of Life (Constable and Co., London, 1914)

7. S.V. Petoukhov, V.I. Svirin, L.V. Khazina, Journal of $M$ achinery $M$ anufacture an $d R$ eliability, 44(3), 249-253 (2015)

8. R.V. Jean, Phyllotaxis: A Systematic Study in Plant Morphogenesis (Cambridge University Press, 1994)

9. N.A. B ernstein, Essays on the physiology of movements and the physiology of activity (Moscow, Medicine Publ., 1966).

10. J. M cFadden, J . A 1-Khalili. P roceedings of the Royal Society A, 474(2220), 1-13 (2018)

11. H.F röhlich e ditor, Biological Coherence and Response to External Stimuli (Springer, 1988)

12. H. F röhlich, F . K remer, Coherent Excitations in Biological Systems (Springer-Verlag, 1983)

13. R. Penrose, Shadows of the mind (Oxford University Press, 1994)

14. G.J. Hyland, Electromagn. Med. Biol., 28, 316-329 (2009)

15. V.N. K adantsev, A.N. Goltsov, R ussian t echnolog. journal, 6(2) (2018)

16. I.V. Lundholm, H. Rodilla, W. Wahlgren, A. Duelli, G. B ourenkov, J . V ukusic, R . F riedman, J . S take, T. Schneider, Structural Dynamics, 2(5) (2015)

17. A.R. V asconcellos, F .S. V annucchi, S. Mascarenhas, R. Luzzi, Information, 3(4) (2012) 
18. S.V. Petoukhov, Biosystems, 139 (January 2016)

19. R. Schumann, On Music and Musicians, ed. Konrad Wolff (New York, Pantheon, 1969)

20. S.V.P etoukhov, M .H e, Symmetrical Analysis Techniques for Genetic Systems and Bioinformatics: Advanced Patterns and Applications (IGI G lobal, USA, 2010) 